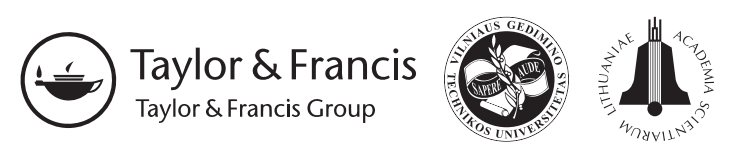

\title{
RSU LOCALIZATION MODEL AND SIMULATION OPTIMIZATION FOR VII NETWORK
}

\author{
Jian Sun ${ }^{1}$, Yuwei Yang ${ }^{2}$ \\ Dept of Traffic Engineering, Tongji University, No 4800, Cao'an road, 201804 Shanghai, China

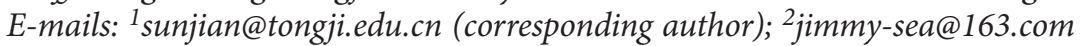

Submitted 9 September 2010; accepted 2 September 2011

\begin{abstract}
While facing the needs for Vehicle Infrastructure Integration (VII) applications in traffic management, the paper deals with the problem of locating Road Side Units (RSU) for VII deployment. After analyzing the difference between traditional problems of locating traffic information detector and the problem of RSU location, a significance ranking model for RSU localization and three kinds of Significance Degree (SD) computing strategies are put forward. A VII simulation environment for the purpose of RSU localization optimization within VISSIM microscopic traffic simulation software is established developing add-on functions using VISSIM's Component Object Model (COM). A VII test bed of the Olympic Park network in Beijing is taken as an example to evaluate the performance of RSU localization model. The results of simulation experiments indicate that the mixed SD strategy considering both speed and route monitoring is superior to the other two SD strategies. Then, the impact of RSU number and OBE market penetration rate on the evaluation measures of traffic monitoring are studied with reference to the proposed mixed SD strategy. In this case, the evaluation measures of optimized RSU configurations generated by the ranking algorithm are always better than those of random RSU configurations. In addition, the benefits of optimized RSU configurations increase along with RSU number and market penetration rate while the benefits of random RSU configurations are more fluctuant.
\end{abstract}

Keywords: VII, traffic management, RSU localization, significance ranking, simulation evaluation, VISSIM.

\section{Introduction}

Over the past decade, the rapid development and application of electronic information and wireless communication technologies have promoted the continued deployment of Vehicle Infrastructure Integration (VII) based on vehicle-vehicle and vehicle-infrastructure communications. Due to the possibilities of reducing the time of driver's reaction, the capability of VII to enhance traffic mobility and safety has been demonstrated drawing considerable attention from governments, enterprises and research institutions. The major VII projects (now called IntelliDrive in the US) have been initiated in California, Michigan and Arizona (Amanna 2009). The Ministry of Science and Technology of China has emphasized VII as one of the key research areas in the national science and technology plan, and is planning to establish VII test beds in Beijing and Shanghai.

Several efforts are being undertaken for the evaluation of VII safety applications while other endeavours are focused on VII applications in traffic management (Oh et al. 2010; Li et al. 2008). VII is expected to provide transportation managers with a complete awareness of motor vehicle movements by detecting probe vehicles equipped with on-board equipment (OBE) and enabling monitoring traffic network states. However, many factors affect the quality and quantity of probe vehicle data offered by VII; these data include market penetration rate, the generation and uploading process of VII snapshots, communication delays, roadside unit (RSU) configurations (i.e. the number and position of RSUs), RSU communication range ( $\mathrm{Li}$ et al. 2008; Shladover et al. 2008) etc. The placement of RSUs in a network is one of the most important considerations for VII deployment because this poses significant implications for VII traffic monitoring applications. However, in many existing VII test beds, the locations of RSUs are determined by empirical analysis, and only a few studies have focused on RSU location optimization (Amanna 2009; Tanikella et al. 2007a, 2007b).

This paper describes the second stage of research effort aimed at developing an RSU localization model and simulation optimization method. The objectives of this research are to:

- address the needs of urban road network traffic management and investigate an RSU localization model based on VII functions; 
- develop a virtual VII test bed within VISSIM microscopic traffic simulation software and evaluate the proposed RSU localization model to overcome limitations associated with real-world test beds;

- explore the effects of different parameters of RSU localization model on signalized networks.

The remainder of this paper is organized as follows. Section 2 reviews previous research on facility localization problems. Section 3 describes the proposed RSU localization model and simulation optimization method. Section 4 illustrates the establishment of a VII simulation environment. A VII test bed is taken as a case study to evaluate the performance of the RSU localization model in Section 5. Section 6 summarizes research and draws conclusions.

\section{Research Review}

Traditional traffic detection includes fixed-point traffic detection typified by induction loop, infrared and microwave radar detection methods as well as by detection techniques for automatic vehicle identification (AVI).

The localization of a fixed-point traffic detector has been a popular issue since traffic detection has been widely applied. Therefore, several methods such as empirical analysis, OD matrix estimation and travel time estimation methods have been put forward (Cipriani et al. 2006; Ehlert et al. 2006; Yang et al. 2006).

The AVI location problem is generally investigated in relation to two purposes one of which is travel time estimation and another is OD estimation. Arafeh and Rakha (2005) formulated the AVI location problem as a quadratic $0-1$ optimization problem, in which objective function parameters represent benefit factors that capture travel time variability along the specified trips; the authors then developed a genetic algorithm to solve this problem. Sherali et al. (2006) offered an algorithm for optimally locating AVI tag readers by maximizing benefits accumulated from measuring travel times on a transportation network. He also wanted to improve the accuracy of OD estimation by covering as many OD pairs as possible. Teodorovic et al. (2002) attempted to solve the AVI equipment location problem as a multiobjective optimization problem by building a mixedobjective function considering both the number of AVI equipment and covered OD pairs. A genetic algorithm was used to solve the proposed model with static path information.

Research on the VII proof-of-concept reveals that vehicle-to-infrastructure communication is enabled using the dedicated short range communication technique. Probe vehicles are equipped with OBE store data regarding running information of the vehicles until they come within the range of RSU. The stored snapshots are then assumed to be completely and simultaneously transmitted. The application of collecting probe vehicle data in traffic management enables traffic managers to monitor traffic flow in large-scale networks. VII requires RSUs to be placed at key locations to enable VII-equipped vehi- cles to communicate with RSUs and transfer probe vehicle data to applications (Tanikella et al. 2007a, 2007b).

The problem of RSU location differs from those of fixed-point traffic detectors and AVI readers mainly in the following aspects:

- In the VII network, RSU coverage includes direct and indirect coverage made possible by snapshot data. This feature enables capturing traffic flow information on links beyond the range of RSU communication. However, data transmission is accomplished with some time lag.

- The quality and quantity of probe vehicle data that a RSU can receive is generally associated with the location of the nearby RSU because the distance between two adjacent RSUs on a specific path affects the process of collecting probe data on VII-equipped vehicles.

- Differently from the problems of the fixed-point detector and AVI reader location, solving the problems of RSU location using the analytical approach is a hard task because of the complicated snapshot process and inherent features of the VII system.

Using the above analysis as a basis, we propose a RSU localization ranking model for VII traffic monitoring applications. A simulation optimization method for RSU location is also investigated on the basis of the established VII simulation environment for evaluating RSU localization.

\section{Optimization of RSU Location Simulation}

The current version of VII architecture provides preliminary information on the rules of RSU placement: RSUs are to be uniformly spaced along the road. The underlying philosophy, which is an empirical approach, informs that higher-speed roads require fewer RSUs than do lower-speed facilities (Farradyne 2005). The number and placement of RSUs in a road network are the major factors influencing the benefits obtained from VII applications; thus, determining appropriate RSU configuration is necessary to maximize the advantages of VII application.

The paper proposes an RSU ranking algorithm (RSURA) for RSU location problems arising in urban networks. The underlying idea of RSURA is to locate RSUs at key points with a high significance degree (SD) after determining the SD value of every optional RSU location. Given that RSUs are envisioned to serve a large number of intersection safety applications, RSUs would be located at major arterial intersections by default. Thus, optional RSU locations are assumed to involve all nodes in a network and intermediate points of links longer than twice the length of RSU communication range. In a given road network, traffic monitoring evaluation measures are calculated after the simulation of the initial fully-located RSU configuration, in which RSUs are located at all optional points determined before simulation. At a later stage, new RSU configuration is generated by disabling one RSU of the initial full-scale RSU 
configuration. The defined traffic monitoring evaluation measures are calculated in the same manner after another simulation. By comparing the deviations between the evaluation measures of the initial configuration and those of the new one, the SD of the point where disabled RSU is located is computed. From this point, the SD of every optional RSU location point can be calculated. Because an SD list of optional points has been generated, a sequence of optional points is arranged in descending SD order. To obtain possible near-optimal RSU configuration with a given number of RSU, RSUs should be located at the most 'important' optional points, in which the computed SD are relatively higher than other optional points.

The description above shows that the SD computing strategy is the key to RSURA, to ensure the effectiveness of which an appropriate SD strategy must first be defined. Let $\mathrm{R}$ be the initial full-scale RSU set where $|R|$ is the number of RSUs in set $R$, and $\left|\bar{R}_{i}\right|$ is a subset of $R$ excluding the $i_{\text {th }}$ RSU, $i=1,2, \ldots, i, \ldots,|R|$. Thus, every set $\left|\bar{R}_{i}\right|(i=1,2, \ldots, i, \ldots,|R|)$ contains $|R-1|$ RSUs. Let $\left|S D_{i}\right|$ denotes the SD of the optional point where the $i_{\text {th }}$ RSU in $R$ is located, and $\left|M A E_{\bar{R}_{i}}\right|$ denotes traffic monitoring evaluation measure [mean absolute error (MAE) for mean link speed] for RSU configuration $\left|\bar{R}_{i}\right|$. Similarly, let $\left|M A E_{R}\right|,\left|R I_{\bar{R}_{i}}\right|$, and $\left|R I_{R}\right|$ be evaluation measures for the corresponding RSU configuration [Route Integrity (RI), the proportion of vehicle routes detected by RSUs]. Three preliminary SD strategies are designed as follows:

$$
\begin{gathered}
\text { Stra 1: } S D_{i}=\left|M A E_{\overline{R_{i}}}-M A E_{R}\right|, \\
i=1,2, \ldots, i, \ldots|R| . \\
\text { Stra 2: } S D_{i}=\left|R I_{\overline{R_{i}}}-R I_{R}\right|, \\
i=1,2, \ldots, i, \ldots|R| . \\
\text { Stra 3: } S D_{i}=\left|\frac{M A E_{\overline{R_{i}}}}{R I_{\overline{R_{i}}}}-\frac{M A E_{R}}{R I_{R}}\right|, \\
\quad i=1,2, \ldots, i, \ldots|R| .
\end{gathered}
$$

Strategy 1 considers link performance measurements, Strategy 2 considers OD monitoring and Strategy 3 (also called mixed SD strategy) considers both speed and route factors. The process of RSURA is described as follows:

Step 1: Without the constraint of RSU number, the initial fully-scale RSU configuration set $R$ is determined where RSUs are located at all optional locations.

Step 2: $|R+1|$ simulations are run for RSU sets $\mathrm{R}$ and $\left|\bar{R}_{i}\right|(i=1,2, \ldots, i, \ldots,|R|)$ to calculate VII application evaluation measures of each set.

Step 3: An SD computing strategy is defined, considering the application targets of VII network. (e.g., enhancing traffic safety or improving the quality of traffic monitoring data).

Step 4: The defined SD is calculated for every optional location point in set $R$.

Step 5: A sequence of optional points is generated in descending SD order. For the given RSU number $\left|R^{*}\right|\left(\left|R^{*}\right|<|R|\right)$, the RSUs of the proposed nearoptimal set $\mid R^{*}$ should be located on the top of $R^{*}$ points in this sequence.

Step 6: The simulation of possible near-optimal RSU configuration $\mid R^{*}$ is conducted, and the corresponding VII application evaluation measures are output.

Step 7: If $R^{*}$ satisfies demand for designed VII applications, the loop is terminated; otherwise, we return to Step 3.

\section{VII Simulation Environments}

Although field evaluations are highly sought, test bed deployments often come with high costs. This imposes big constraints on the ability to assess the full potential of proposed VII applications through field evaluations (Dion et al. 2009).

In this paper, a VII simulation environment for RSU location evaluation within VISSIM microscopic traffic simulation software is established. This simulation environment is applied to the RSURA process to calculate the effectiveness of the proposed algorithm. The VII simulation environment based on the physical architecture of VII deployment is developed by the Federal Highway Administration (FHWA) and includes four functional modules: VII communication, microscopic traffic, strategy application and database modules. A high level overviewing the architecture of RSU location simulation is displayed in Fig. 1.

\subsection{Communication Module}

VII communication module is the key component of VII simulation environment representing the entire overlay of VII communication network in real-world applications, including OBUs, RSUs and VII message switches. VII communication module emulates the infrastructure of VII communications at a functional level to transmit data on traffic simulator to the strategy application module via the function of collecting a snapshot of a probe vehicle. Detailed technical simulations of communications such as signal attenuation, signal masking, and communication delay are not considered in detail. The function of collecting a snapshot of a probe vehicle is implemented via programming according to the operational concepts outlined in surface vehicle standard SAE J2735-2009 and studies on data regarding a snapshot of a probe vehicle (Dion et al. 2009; Tanikella et al. 2007a, 2007b). The function of collecting a snapshot of a probe vehicle includes two processes described as follows.

The process of snapshot generation:

- Two types of snapshots are generated while a vehicle is out of the communication range of any RSU: periodic snapshots taken at predefined intervals and start/stop snapshots taken when a 


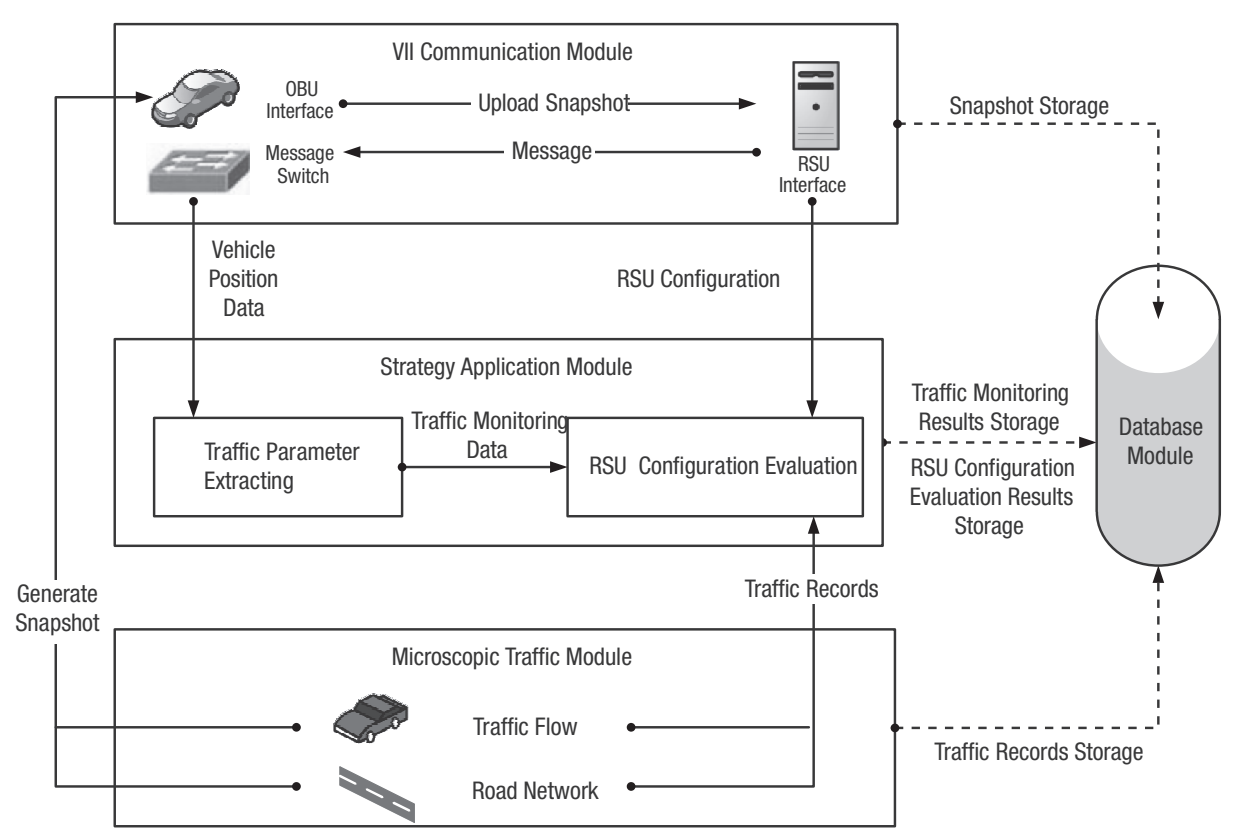

Fig. 1. The architecture of RSU location simulation

vehicle is assumed to have stopped or started to move following a stop.

- Periodic snapshots are generated at intervals based on the speed of a vehicle.

- A stop event snapshot is recorded after a vehicle has remained stationary for 5 seconds since the last recorded stop. Start event snapshots are generated for vehicles at a stationary state when reaching a speed threshold of $16 \mathrm{~km} / \mathrm{h}(10 \mathrm{mph})$.

The process of snapshot storage and upload:

- Snapshots are inserted in the buffer of a vehicle while it is out of the communication range of any RSU.

- When communication is established with an RSU, all snapshots stored in the buffer of the vehicle are assumed uploaded to the RSU in single transmission with no delay.

- Periodic and start/stop snapshots are added to the buffer as long as there is space available. FIFO strategy is adopted for snapshot buffer management. When the buffer is full, the second oldest available periodic snapshot is removed to make room for a new snapshot.

\subsection{Microscopic Traffic Module}

The microscopic traffic module provides a description of an actual road network, along with control systems and vehicles traversing the road network. During the simulation process, data on individual vehicles are collected for generating a snapshot of a probe vehicle applying VII communication module. Traffic records are sent to the module of strategy application as reference data for evaluating RSU configuration. Widely used VISSIM 5.2 is adopted as traffic simulator (VISSIM 5.20: User Manual 2009). To dynamically interact with the simulator and obtain the current data considering RSU configuration, the component object model (COM) provided by VISSIM is used.
A snapshot records information on the position of each vehicle in the road network. Snapshot records are collected at every simulation second for 'benchmark' data and aggregated at every pre-defined interval for 'field-collected data'. A standard format of snapshot data (Table 1) is defined including all parameters taken from dataset standard SAE J2735-2009.

Table 1. The format of snapshot data

\begin{tabular}{|c|c|}
\hline Vehicle ID & $\begin{array}{c}\text { ID of the vehicle that generates the } \\
\text { snapshot }\end{array}$ \\
\hline Time (s) & $\begin{array}{l}\text { The second simulation when the snapshot } \\
\text { is generated }\end{array}$ \\
\hline Current link ID & The current link for travelling vehicles \\
\hline VII vehicle & $\begin{array}{l}\text { If the vehicle has an OBE, this Boolean } \\
\text { variable will be true }\end{array}$ \\
\hline Latitude & The current latitude of a vehicle \\
\hline Longitude & The current longitude of a vehicle \\
\hline Altitude (m) & The current altitude of a vehicle \\
\hline Speed $(\mathrm{km} / \mathrm{h})$ & The current speed of a vehicle \\
\hline $\begin{array}{l}\text { Current vehicle } \\
\text { state }\end{array}$ & $\begin{array}{l}\text { Record the current state of a vehicle: } \\
\text { running, stopped or starting }\end{array}$ \\
\hline
\end{tabular}

\subsection{Strategy Application Module}

The module of strategy application implements external functions supported by VII, which uses information generated by the simulator and that obtained from VII communication module to obtain traffic monitoring results and evaluate the advantages of the given RSU locations. These tasks are accomplished by assessing the accuracy of traffic monitoring measures. The strategy application module consists of two sub-modules: traffic parameter extracting a sub-module for traffic state 
monitoring and a sub-module for evaluating the benefit of RSU configuration.

The purpose of traffic parameter extracting the sub-module is to obtain detected data related to the state of traffic and covering volume, speed, occupancy, origin-destination matrix and vehicle routes. Mean link travel speeds and probe vehicle routes are chosen at the preliminary research stage while other traffic state parameters can be used for future research. The speed of mean link travel is calculated, considering that each probe vehicle can provide multiple instantaneous speed samples while in a link. For all probe vehicles in a link during one-minute reporting interval, the mean travel speed of this link in the interval is computed ( $\mathrm{Li}$ et al. 2008). In routes, probe vehicle routes involving link ID, which a probe vehicle has traversed, are recorded based on information provided by VII communication module. In general, because of snapshot loss during storage in a buffer, the obtained probe vehicle routes are incomplete in a network with low RSU density.

The purpose of the sub-module for evaluating the benefit of RSU configuration is to identify the range of errors associated with the estimates of traffic state parameters computed by other sub-modules having different RSU configurations and simulation scenarios. Three measures, namely, MAE for mean link travel speed, probe vehicle route integrity (RI) and traffic monitoring coverage (TMC) are considered in the evaluation of RSU configurations, as defined in (Tanikella et al. 2007a, 2007b):

$$
M A E=\frac{1}{L A} \sum_{a=1}^{A} \sum_{l=1}^{L}\left|V_{l a}-V_{l a}^{*}\right| .
$$

Let $A$ be the number of speed reporting intervals during simulation, and $L$ - the number of links with traffic data reports. Let $V_{l a}$ denotes the mean link travel speed of link $l_{\text {th }}$ during reporting interval $a_{\text {th }}$ that is computed based on uploaded data on a probe vehicle and $V_{l a}^{*}$ denotes the reference value of mean link travel speed computed from traffic simulator records. The estimation of $V_{l a}$ is cited from (Li et al. 2008).

$$
R I=\frac{1}{B} \sum_{b=1}^{B} \frac{1}{N_{b}} \sum_{n_{b}=1}^{N_{b}} \frac{S_{n_{b}}}{S_{n_{b}}^{*}} .
$$

Let $B$ denotes the number of route reporting intervals, $N_{b}$ - the number of probe vehicles that uploaded snapshots to RSUs during reporting interval $b_{\text {th }}$, $S_{n_{b}}$ - the number of links detected by VII network in which the $n_{\text {th }}$ probe vehicle in Interval $B$ has traversed and $S_{n_{b}}^{*}$ - the traversed link number of the probe vehicle recorded by the simulator in Interval $B$.

$$
T M C=\frac{L}{L^{*}} \cdot 100 \% \text {. }
$$

Symbol $L^{*}$ denotes the total number of links in the given road network.

\subsection{Database Module}

The database module functions as a storage room for three types of data: traffic records from the traffic simulator, probe vehicle snapshots generated by VII communication module and results obtained from the strategy application module.

\section{Case Study}

\subsection{Test Bed}

The Olympic Park network in Beijing is taken as a case study to evaluate the performance of RSU localization model. It is also one of the optional bases of Chinese National VII demonstration project.

In 2008, the microscopic model consisting of 169 zones and 873 links was calibrated according to field surveys (Fig. 2). The simulation model exports $100 \mathrm{sec}-$

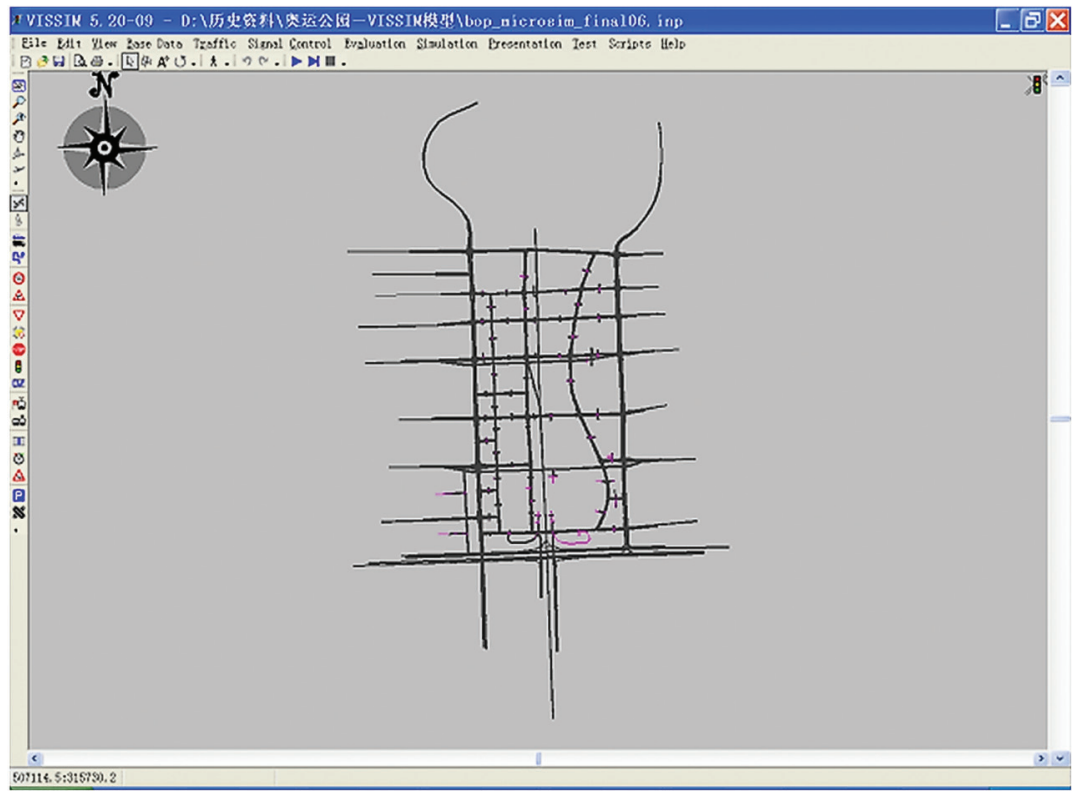

Fig. 2. The test network of Beijing Olympic Park 
tion traffic volumes compared with field measurement ones. The average relative error of the volume is $10 \%$ and the average relative error of travel time is $12 \%$. Therefore, the accuracy of the model satisfies requirements for test application.

\subsection{Simulation Experiments}

Before simulation experiments, 'full-scale' RSU configuration $R$ has been determined with 10 RSUs located at 10 optional arterial intersections of the Olympic Park road network. Regarding such configuration, the number of RSUs is dependent on both traffic condition and the mechanism of the process of collecting VII data. In this case, we choose this number based on the traffic condition of the peak hour so that 'full-scale' configuration can generate accurate enough traffic data under different situations. To obtain fundamental results used for computing three types of designed SD values of each optional points, 11 simulation scenarios along with RSU configurations of $R, \bar{R}_{1}, \bar{R}_{2}, \ldots$, and $\bar{R}_{10}$ are adopted for multiple simulations.

For each scenario, 8 simulation experiments were run by changing random seeds to take care of bias data and the values of 3 measures were the average value of 8 samples. Each experiment is simulated for one hour, having 40157 vehicles processed through the network. To account for network loading effects and ensure that the equilibrium state has been reached, a 30-minute warm-up is initiated before starting the one-hour evaluation period. Aside from RSU locations, other VII parameters (Table 2) are determined before simulations taking into account 11 scenarios (SAE J2735-2009; VII Probe Message Processes... 2006).

Table 2. Simulation parameters

\begin{tabular}{lc}
\hline \multicolumn{1}{c}{ Variable } & Value \\
\hline RSU communication range $(\mathrm{m})$ & 200 \\
\hline A low interval of snapshot generation $(\mathrm{s})$ & 4 \\
\hline A high interval of snapshot generation $(\mathrm{s})$ & 20 \\
\hline $\begin{array}{l}\text { Speed threshold of snapshot generation at a low } \\
\text { interval }(\mathrm{km} / \mathrm{h})\end{array}$ & 32 \\
\hline $\begin{array}{l}\text { Speed threshold of snapshot generation at a high } \\
\text { interval }(\mathrm{km} / \mathrm{h})\end{array}$ & 96 \\
\hline $\begin{array}{l}\text { The size of snapshot buffer } \\
\text { The market penetration rate of VII vehicle }(\%)\end{array}$ & 100 \\
\hline Speed threshold of start event $(\mathrm{km} / \mathrm{h})$ & 16 \\
\hline Timer threshold of stop event $(\mathrm{s})$ & 5 \\
\hline Snapshot record while stopped & False \\
\hline
\end{tabular}

\subsection{Results Analysis}

\subsubsection{Analysis of RSU SD Strategy}

The strategy of RSU SD is the key of RSURA. Therefore, considering VII traffic monitoring applications, investigating the effects of SD strategies on the advantages of RSU configurations is essential for determining an op-

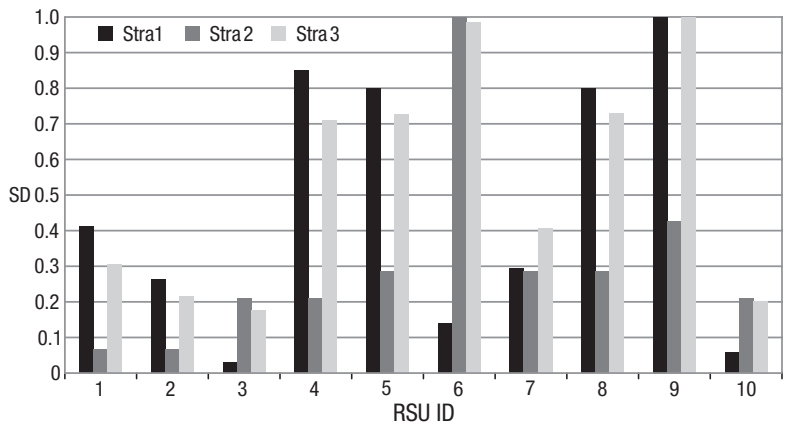

Fig. 3. SD results using three different SD strategies

timum SD strategy for RSURA. Three SD strategies defined in Section 3 are used. Three SD values of each RSU in set $R$ are computed based on fundamental simulation results. Calculation results generated after normalization are illustrated in Fig. 3.

$\mathrm{SD}$ values under different strategies vary from one another for each RSU. Three sequences of 10 RSUs in descending SD order are also different and lead to different results of RSU location considering RSURA for a given RSU number. For example, RSU 4 ranks 9 in the first RSU sequence based on SD strategy 1, 4-in the second sequence and 6 - in the third one.

The proposed SD strategies have been proved varying; thus, determining their suitability for solving the problem of RSU location becomes necessary. To investigate the effects of different SD strategies on RSURA effectiveness, we generate 30 RSU configurations. For a given number of RSUs varying from 1 to 10 in steps of one, three RSU configurations are generated by RSURA using three SD strategies. Then, another group of simulation experiments is conducted to obtain the measures for evaluating each scenario (Figs $4 a-c$ ).

Fig. $4 \mathrm{a}$ indicates that the MAE measures of RSU configurations based on three SD strategies are close to one another when the RSU number is above six. However, Strategy 1 behaves better than the other two up to $1 \mathrm{~km} / \mathrm{h}$ on MAE when the RSU number is below five. Overall, no significant difference in speed monitoring effect is found for three presented strategies, because differences between them in speed monitoring are related to the total number of speed samples available for speed computation of mean link travel. The sample numbers of the strategies are virtually identical to the RSU number, VII market penetration rate, buffer size and the loss ratio of snapshots.

Fig. $4 \mathrm{~b}$ shows that monitored probe vehicle RI for simplified SD Strategy 2 and mixed SD Strategy 3 are always at the same level taking into account different RSU numbers. The results of Strategy 1 are lower than those of the other two with a maximum difference of $15 \%$.

Fig. $4 \mathrm{c}$ displays that mixed SD Strategy 3 constantly delivers more efficient performance than the other two up to $9 \%$ on TMC only when the RSU number is less than four. For all three strategies, TMC nonlinearly increase along with RSU number, indicating that during VII deployment, the advantages presented by each RSU possibly decrease with increasing the RSU number. 
a)

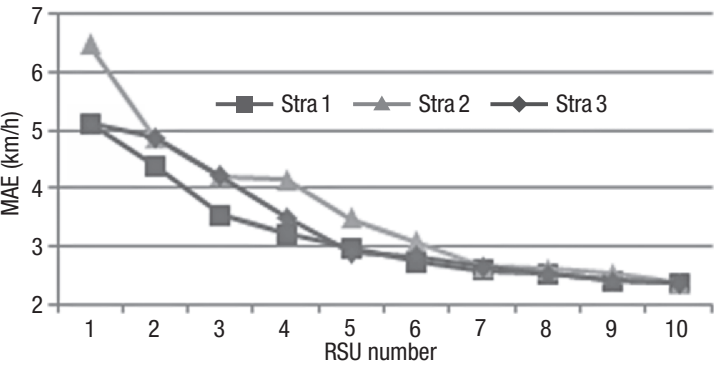

b)

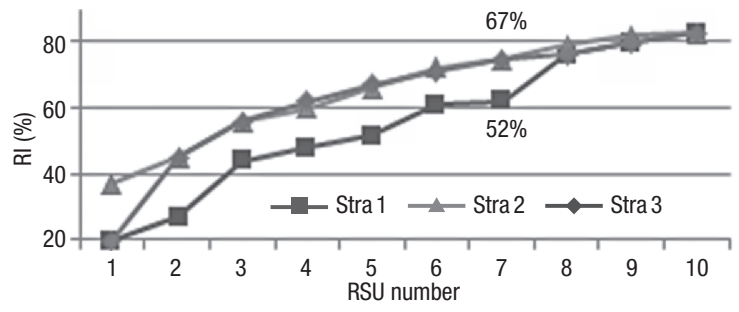

c)

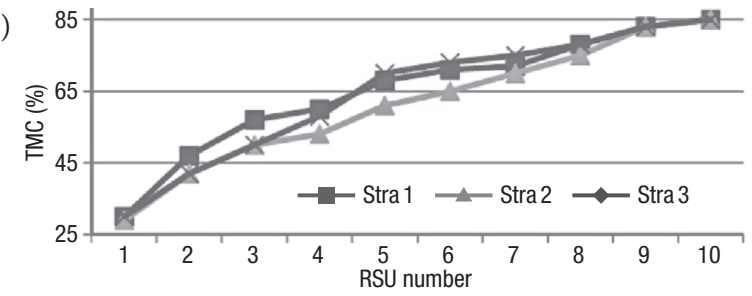

Fig. 4. Different measure changing trends with RSU number: a - MAE; b - RI; c - TMC

Simulation results show that different SD strategies have a significant influence on the effectiveness of RSURA. The above analysis discloses that the mixed SD strategy has been proved suitable for solving the problem of RSU location and is adopted in RSURA for studies conducted in Section 5.3.2.

\subsubsection{Analysis of RSURA Sensitivity}

Because the percentages of the equipped OBU vehicle are quite low at the early stages of VII deployment, the effects of the market penetration rate on the benefits obtained from RSU configuration have drawn considerable attention from decision makers. To comprehensively evaluate the effectiveness of RSURA and the effects of VII penetration rate of the vehicle market, two series of simulation scenarios are designed. These scenarios are a set of RSURA-based RSU configurations (generated by RSURA) called 'Series Ranking' and a set of configurations (generated by randomly selecting RSU locations) based on the random choice method 'Series Random'. For each series, other simulation parameters are based on the set of parameters listed in Table 2; the penetration rate of VII market is modified from 5\% to $100 \%$, whereas the RSU number varies from 1 to 10 . For each scenario, a one-hour simulation experiment is conducted. The obtained results are shown in Figs $5 \mathrm{a}-\mathrm{c}$.

Fig. 5a illustrates the simulation results of MAE for estimating mean link travel speeds under two series of simulation scenarios, including changes in market penetration rates and RSU numbers. The obtained results show that the MAE of Series Ranking illustrated by the lower surf is always better than that of Series Random illustrated by the higher surf up to $1.67 \mathrm{~km} / \mathrm{h}$. From the results of Series Ranking, MAE minimally changes with an increase in the market penetration rate when the RSU number is small. However, MAE significantly improves with increasing the market penetration rate when the RSU number is relatively large. This result can be useful for VII deployment as the performance of system operation is improved first investing sufficient RSUs and then increasing the percentage of vehicles equipped with OBU. Conversely, a very limited decrease in the estimation error of mean link travel speed occurs for the market penetration rate exceeding $40 \%$.

a)

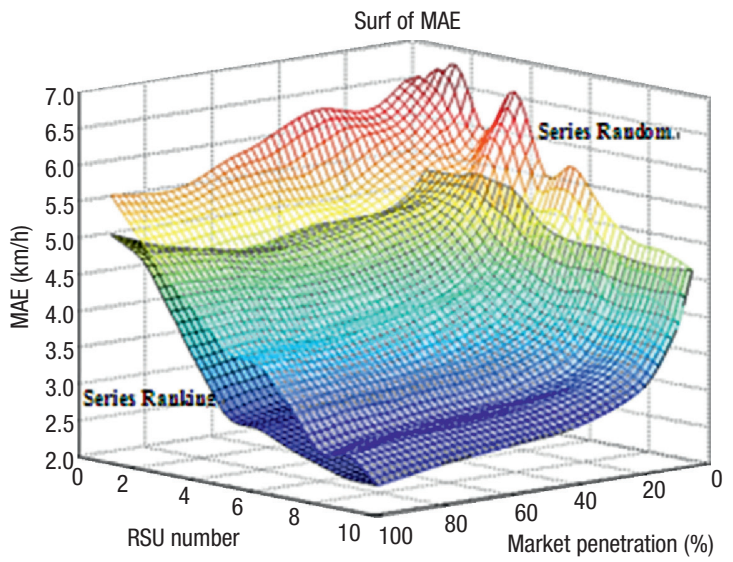

b)

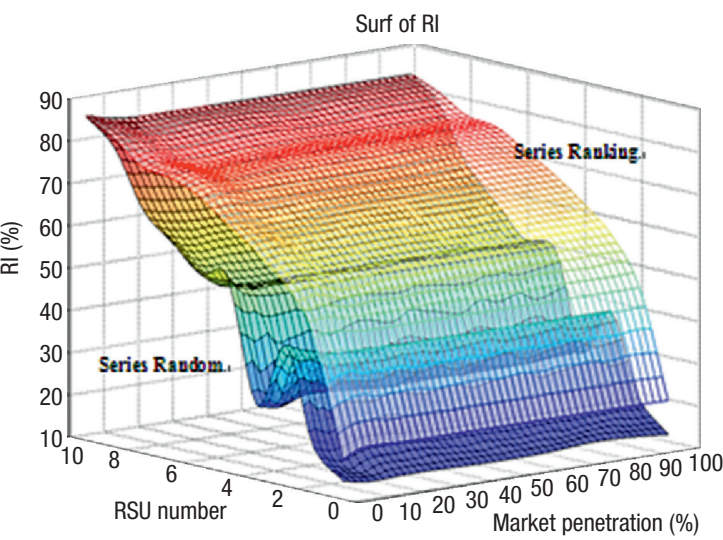

c)

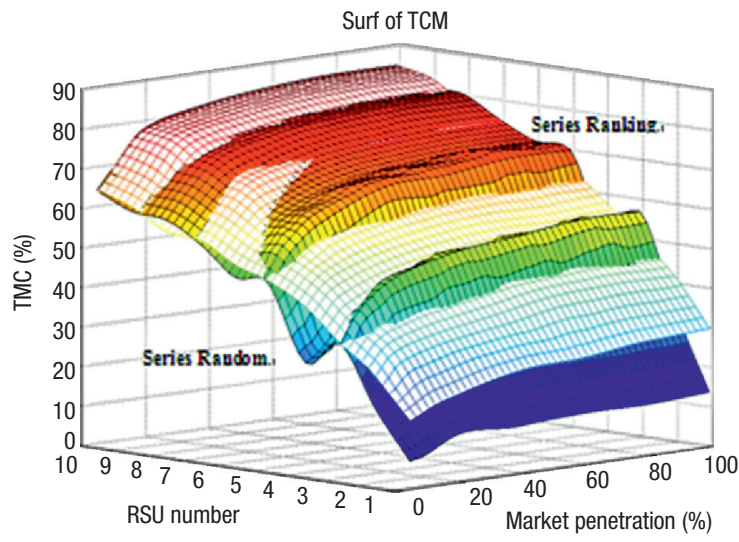

Fig. 5. Effects of the RSU number and market penetration on different measures: $a$ - MAE; b - RI; c - TMC 
Fig. $5 \mathrm{~b}$ shows the simulation results of probe vehicle RI. As expected, more information about probe vehicle routes can be obtained by RSUs located according to RSURA compared with randomly located RSUs. The average difference in RI between Series Ranking and Series Random is as high as $16 \%$. The probe vehicle RI of Series Ranking always increases with the RSU number, whereas Series Random reflects a fluctuating trend. In addition, the market penetration rate has no impact on probe vehicle RI because only the route of a probe vehicle can be obtained by VII. This measure is computed based on the totality of probe vehicles.

Fig. $5 \mathrm{c}$ indicates that the specific results of TMC vary depending on the RSU number and are independent of the location of RSUs in the network because the TMC measures of two series of RSU configurations are at the same level on the whole. A considerable improvement in TMC occurs for market penetration rates under $20 \%$.

From the analysis above, we can conclude that the benefits of optimized RSU configurations generated by RSURA improve with increasing the RSU number and market penetration rate, whereas the benefits obtained from random RSU configurations fluctuate. Effective RSU configuration can be generated by RSURA to improve the quality and quantity of monitoring VII traffic data. The model of RSU localization and the method of simulation optimization proposed in this paper can serve as theoretical and methodological support for RSU location in VII deployment.

\section{Conclusions and Recommendations}

The paper investigated the problem of RSU location for VII deployment addressing the needs of VII applications in traffic management. The article proposed a ranking model for RSU localization and three kinds of SD computing strategies. Additionally, a VII simulation environment for evaluating RSU localization within VISSIM microscopic traffic simulation software was established by developing add-on functions using VISSIM's COM.

The paper described the research effort aimed at developing the RSU localization model and simulation optimization method. Subsequent research can focus on the following aspects:

- investigating the effects of different communication ranges and snapshot generation strategies on RSU configuration benefits;

- designing more reasonable SD strategies for different VII applications to satisfy the requirements of different application scenarios;

- expanding the functions of simulation environments to comprehensively evaluate the performance of VII system.

- taking the probability of RSU malfunction into account to enhance the RSU Ranking Algorithm using Monte Carlo simulation method.

\section{Acknowledgement}

The authors are very grateful to anonymous referees for their insightful and constructive comments and suggestions that have led to an improved version of this paper. This work was supported by the Natural Science Foundation of China $(61004113,50948056)$.

\section{References}

Amanna, A. 2009. Overview of IntelliDrive / Vehicle Infrastructure Integration (VII). Virginia Tech Transportation Institute (VTTI). 11 p. Available from Internet: <http://filebox. vt.edu/users/aamanna/web\%20page/VII-IntelliDrive\%20 Report-edited.pdf $>$.

Arafeh, M.; Rakha, H. 2005. Genetic algorithm approach for locating automatic vehicle identification readers, in Proceedings of IEEE International Conference on Intelligent Transportation Systems - 2005. 13-15 September 2005, 314-319. doi:10.1109/ITSC.2005.1520214

Cipriani, E.; Fusco, G.; Gori, S.; Petrelli, M. 2006. Heuristic methods for the optimal location of road traffic monitoring, in Proceedings of IEEE International Conference on Intelligent Transportation Systems - 2006. 17-20 September 2006, 1072-1077. doi:10.1109/ITSC.2006.1707364

Dion, F.; Oh, J.-S.; Robinson, R. 2009. VII Test Bed Simulation Framework for Vehicle-to-Infrastructure Communication and Application Evaluations, in TRB 88th Annual Meeting Compendium of Papers DVD. 11-15 January 2009, Washington, D.C. $18 \mathrm{p}$.

Ehlert, A.; Bell, M. G. H.; Grosso, S. 2006. The optimisation of traffic count locations in road networks, Transportation Research Part B: Methodological 40(6): 460-479. doi:10.1016/j.trb.2005.06.001

Farradyne, P. B. 2005. Vehicle Infrastructure Integration (VII): VII Architecture and Functional Requirements: Version 1.1. U.S. Department of Transportation. 82 p. Available from Internet: <http://www.vehicle-infrastructure.org/documents/VII\%20Architecture\%20version\%201\%201\%20 2005_07_20.pdf>.

Li, M.; Zou, Z.-J.; Bu, F.; Zhang, W.-B. 2008. Application of vehicle infrastructure integration data on real-time arterial performance measurements, in TRB 87th Annual Meeting Compendium of Papers DVD. 13-17 January 2008, Washington, D.C. 19 p.

Oh, C.; Kim, T.; Kim, W.; Hong, S.; Park, J. 2010. Capabilityenhanced probe vehicle surveillance system with vehicleto-vehicle communications: framework and evaluation, Transportation Research Record 2189: 8-16. doi:10.3141/2189-02

SAE J2735-2009. Dedicated Short Range Communications (DSRC) Message Set Dictionary.

Sherali, H. D.; Desai, J.; Rakha, H. 2006. A discrete optimization approach for locating Automatic Vehicle Identification readers for the provision of roadway travel times, Transportation Research Part B: Methodological 40(10): 857-871. doi:10.1016/j.trb.2005.11.003

Shladover, S. E.; Kuhn, T. M. 2008. Traffic probe data processing for full-scale deployment of vehicle-infrastructure integration, Transportation Research Record 2086: 115-123. doi:10.3141/2086-14 
Tanikella, H.; Smith, B. L.; Zhang, G.; Park, B.; Guo, J.; Scherer, W. T. 2007a. Development and evaluation of a vehicle-infrastructure integration simulation architecture, Journal of Computing in Civil Engineering 21(6): 434-440. doi:10.1061/(ASCE)0887-3801(2007)21:6(434)

Tanikella, H.; Smith, B. L.; Zhang, G.; Park, B.; Scherer, W. T. 2007b. Simulating operations applications of vehicle infrastructure integration: traffic monitoring case study, Transportation Research Record 2000: 35-43. doi:10.3141/2000-05

Teodorovic, D.; Van Aerde, M.; Zhu, F.; Dion, F. 2002. Genetic algorithms approach to the problem of the automated vehicle identification equipment locations, Journal of Advanced Transportation 36(1): 1-21. doi:10.1002/atr.5670360102

VISSIM 5.20: User Manual. 2009. PTV AG. 576 p.

Yang, H.; Yang, C.; Gan, L. 2006. Models and algorithms for the screen line-based traffic-counting location problems, Computers and Operations Research 33(3): 836-858. doi:10.1016/j.cor.2004.08.011

VII Probe Message Processes, Version 1.0. 2006. Federal Highway Administration (FHWA). U.S. Department of Transportation, Washington, D.C. 